\title{
Early Anticipation of Candidacy for Intra- Arterial Reperfusion Therapy Based on Baseline Clinical Stroke Subtypes: Comparison with Multiparametric MRI Taken within 4.5 Hours from Stroke Onset
}

\author{
Yong-Won Kim ${ }^{a, c}$ Dong-Hun Kang ${ }^{\text {b-d }}$ Yang-Ha Hwang ${ }^{\text {a, }}$, d \\ Yong-Sun Kim ${ }^{\text {b-d }}$ Sung-Pa Park ${ }^{a, d}$ \\ Departments of a Neurology and ${ }^{b}$ Neuroradiology, and ${ }^{c}$ Cerebrovascular Center, \\ Kyungpook National University Hospital, and d School of Medicine, Kyungpook National \\ University, Daegu, Republic of Korea
}

\section{Key Words}

Acute stroke · Thrombolysis · Clinical stroke syndromes · Oxfordshire Community Stroke Project classification · MRI

\begin{abstract}
Background: The decision to proceed with intra-arterial (IA) reperfusion therapy is typically made late in the course of in-hospital treatment for acute ischemic stroke. Early anticipation of candidacy for IA reperfusion therapy based on clinical stroke subtypes would be useful for guiding stroke management. The aim of this study was to investigate the relationship between the clinical Oxfordshire Community Stroke Project (OCSP) classification and MRI results taken within a 4.5-hour time window from stroke onset, with the hypothesis that the persistence of major arterial occlusion and extended ischemic penumbra, key criteria for proceeding with IA reperfusion therapy, would be distinctive between the clinical stroke subtypes. Methods: A total of 161 patients with acute ischemic stroke in the anterior circulation were included in this study. All patients were treated with intravenous alteplase, and MRI scans were performed following alteplase initiation. Prior to treatment, the patients were categorized, based on the OCSP classification scheme, as having total anterior circulation infarcts (TACI), partial anterior circulation infarcts (PACI), or lacunar infarcts (LACI). The relationship between OCSP subtypes, MRI parameters, and clinical variables was analyzed. Results: Overall, 40/161 patients (24.8\%) were candidates for IA rescue reperfusion. With respect to the classification, 30/69 TACI (43.5\%), 6/33 PACI (18.2\%), and 4/59 LACI patients (6.8\%) were candidates ( $p<0.001$ ). Major arterial occlusion was found in 56/161 patients (34.8\%), and 46/69 TACI (66.7\%), 6/33 PACI
\end{abstract}


(18.2\%), and 4/59 LACI patients (6.8\%) had a major arterial occlusion $(\mathrm{p}<0.001)$. A perfusiondiffusion mismatch greater than $20 \%$ was found in $85 / 161$ patients (52.8\%). More specifically, 40/69 TACI (58.0\%), 25/33 PACI (75.8\%), and 20/59 LACI patients (33.9\%) had a perfusion-diffusion mismatch $(p<0.001)$. However, in terms of the total area of mismatch, $66.0 \%$ of patients with ASPECTS DWI-PWI $_{2} \geq 2$ (Alberta Stroke Program Early CT Score) were classified as TACI patients $(p<0.001)$ and of the patients with ASPECTS DWI-PWI $_{23}, 74.3 \%$ were classified as TACI patients ( $p$ 0.001). Relative to candidates for IA rescue reperfusion, the clinical TACI group showed $75.0 \%$ sensitivity, $67.8 \%$ specificity, a positive predictive value of $43.5 \%$, and a negative predictive value of $89.1 \%$. Conclusions: In this study, patients classified as having clinical TACI were significantly more likely to be candidates for IA rescue reperfusion. Additionally, they incurred a higher incidence of persistent major arterial occlusion and had a penumbra area that was significantly larger than normal. Therefore, clinical OCSP can be used as an 'early warning system' for IA reperfusion candidacy, which can allow for advanced preparation of IA therapy and theoretically shorten treatment time and reduce infarction.

Copyright (C) 2013 S. Karger AG, Basel

\section{Introduction}

The Oxfordshire Community Stroke Project (OCSP) scheme is a simple clinical classification tool for acute ischemic stroke that can be conducted quickly at bedside [1]. This classification has a role in defining the distinctive clinical characteristics between the OCSP subtypes, and thus it may guide patient management [1-4].

In the era of reperfusion as a target goal of acute stroke treatment, it would be highly useful to anticipate patients having both major intracranial arterial occlusion and measurable ischemic penumbra based on clinical factors alone because the conversion to intra-arterial (IA) rescue reperfusion therapy could be considered early in stroke management [5-8]. Even moderate success in showing relationships between clinical classification and more definitive radiographic results would benefit the early planning and treatment for acute ischemic stroke. Some studies have examined the relationship between clinical OCSP and radiographic results $[2,3,9,10]$. However, no studies relating OCSP classification and MRI parameters, including diffusion-weighted imaging (DWI) and perfusion-weighted imaging (PWI), have been reported using MRI taken within a 4.5-hour time window from stroke onset. This is significant since evaluating penumbra, a key criterion for IA rescue reperfusion candidacy, requires early MRI.

The aim of this study was to investigate the relationship between clinical OCSP subtypes and MRI results performed within a 4.5-hour time window from stroke onset, with the hypothesis that the persistence of major arterial occlusion and ischemic penumbra would be distinctive between the clinical stroke subtypes. The goal is early anticipation of IA reperfusion candidacy to allow for advanced preparation of IA therapy and theoretically shorten treatment time.

\section{Subjects and Methods}

\section{Patients}

We reviewed consecutive patients from the prospectively collected acute ischemic stroke registry, who had been admitted to the emergency center of our institute within $3 \mathrm{~h}$ of stroke symptom onset between May 2006 and December 2010. Patients with acute ischemic stroke were enrolled based on the following inclusion criteria: (1) treatment in our institution with 
Fig. 1. Simplified diagram of the study. GRE = Gradient-echo imaging.

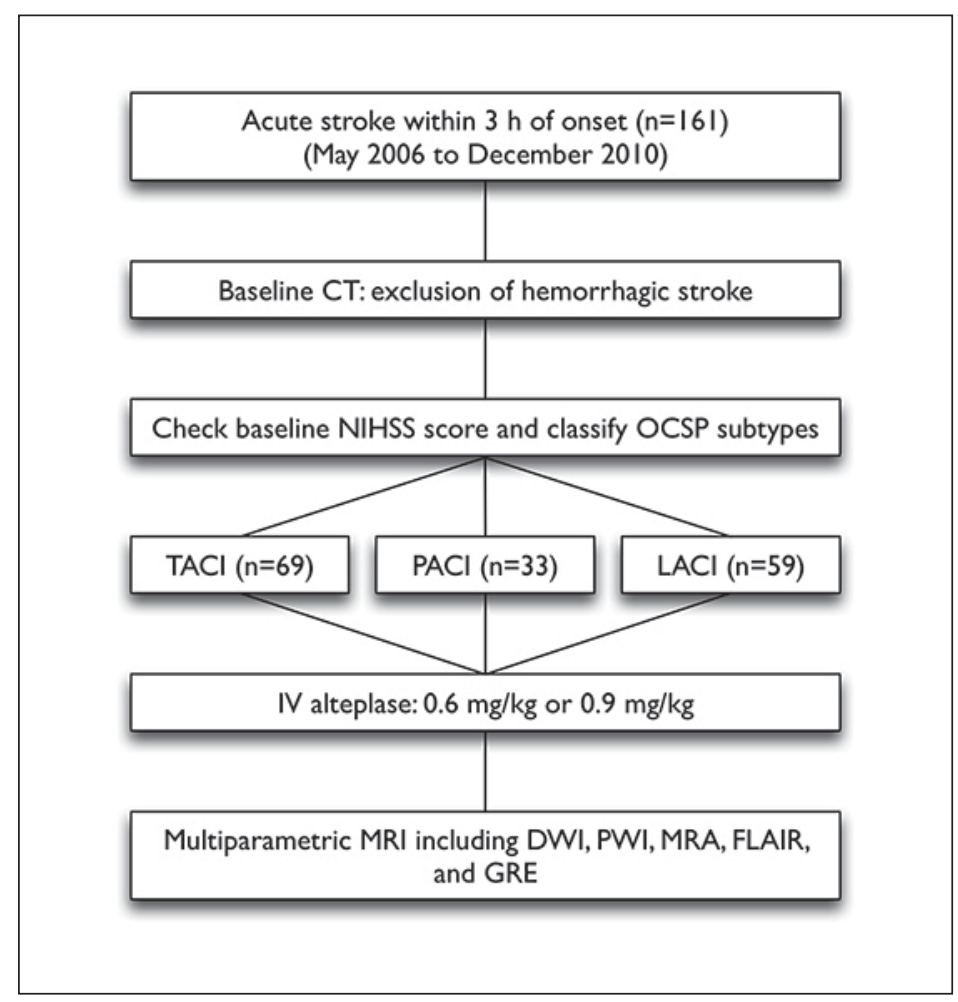

intravenous (IV) alteplase (recombinant tissue plasminogen activator) within a 3-hour time window based on a non-contrast CT scan to rule out hemorrhage; (2) performance of MRI following initiation of alteplase, such that it was within $4.5 \mathrm{~h}$ from stroke onset; (3) confinement of vascular territory of acute ischemic stroke to the anterior circulation, and (4) documentation of informed consent. We excluded patients with posterior circulation stroke because anterior circulation and posterior circulation strokes were thought to be separate entities, with different underlying pathogenesis and natural histories. The local institutional review board approved this study for retrospective analysis.

\section{Clinical Evaluation and Treatment}

All patients were first classified according to the OCSP classification scheme as having total anterior circulation infarcts (TACI), partial anterior circulation infarcts (PACI), posterior circulation infarcts, or lacunar infarcts (LACI) [1]. Prior to IV alteplase infusion, physical and neurological examinations, including the National Institutes of Health Stroke Scale (NIHSS) scoring, were carried out as well. Once patients were classified and scored, the alteplase treatment (a dose of 0.6 or $0.9 \mathrm{mg} / \mathrm{kg}$ determined by the on-call neurologist) was administered after a brain CT had been obtained and hemorrhage was ruled out. We used criteria set by the National Institute of Neurological Disorders and Stroke (NINDS) to determine eligibility for IV alteplase infusion [11]. Patients were not excluded for passing the upper age limit or for exceeding the upper or lower limits on the NIHSS score. A simplified diagram of this study is shown in figure 1.

\section{Interpretation and Classification of MRI}

Multiparametric stroke MRI using a 3.0-T machine (Signa Excite; GE) was performed following initiation of the alteplase infusion. The imaging protocol is described in detail in 


\section{Cerebrovascular \\ Diseases}

Fig. 2. Representative figures for radiographic IA candidates and lacunar infarcts.

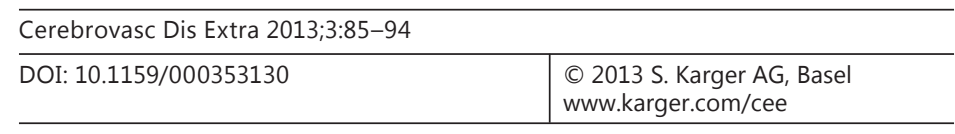

Kim et al.: Baseline Stroke Subtype and Anticipation of Rescue Reperfusion Therapy

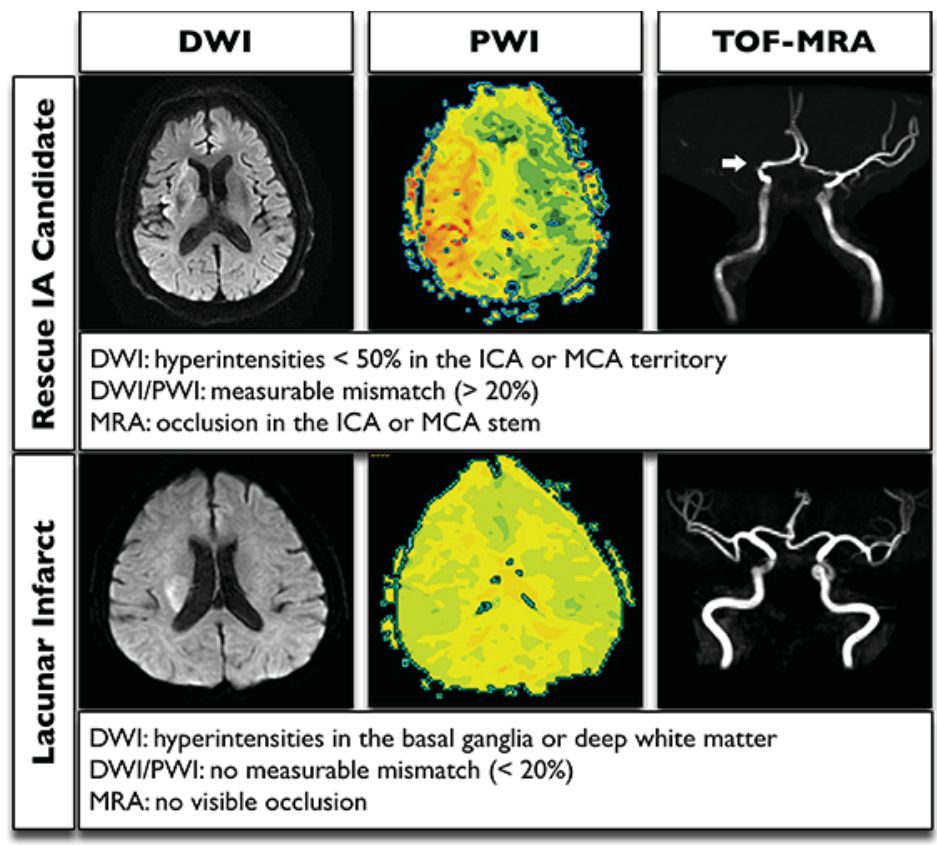

online supplementary table 1 (see www.karger.com/doi/10.1159/000353130). MRI results were independently evaluated by two experts, a neuroradiologist (D.-H.K.) and a neurologist (Y.-W.K.), who were blind to any clinical information. DWI and PWI at baseline were assessed using the Alberta Stroke Program Early CT Score (ASPECTS) to define the extent of ischemic injury and misery perfusion [12]. The presence of arterial occlusion was defined as signal loss, with signal rarefaction of distal branches of major intracranial vessels, observed using three-dimensional time-of-flight MR angiography (3D TOF-MRA) and co-located lesions observed on DWI [13]. A PWI-DWI mismatch was defined as being present if the areas of perfusion abnormalities (a mean transit time delay greater than $4 \mathrm{~s}$ compared with the contralateral side) were more than $20 \%$ larger than those of the diffusion abnormalities on visual assessment. We operationally defined candidacy for IA rescue reperfusion based on three criteria: (1) DWI hyperintensities showing less than $50 \%$ of vascular territory affected (ASPECTS $_{\text {DWI }}>5$ ); (2) PWI-DWI mismatch with a PWI area 20\% larger than the DWI area, and (3) presence of internal carotid artery (ICA) or middle cerebral artery (MCA) stem occlusion on 3D TOF-MRA (fig. 2). We also defined a radiographic lacunar infarct as a spheroidal infarct in the deep white matter or the basal ganglia with a maximum diameter of $15 \mathrm{~mm}$ without mismatch and arterial occlusion (fig. 2). The presence of a hyperintense vessel sign (HVS) was identified as a tubular hyperintense signal, relative to the gray matter, from two or more vessels on fluid-attenuated inversion recovery (FLAIR) imaging [14]. Disagreement between the two MRI raters was resolved by consensus determination.

\section{Statistical Analysis}

The relationship between OCSP subtypes, NIHSS scores, clinical and time variables and MRI parameters was analyzed. Descriptive and frequency statistical analyses were performed using SPSS for Windows (version 19.0, SPSS Inc.). Statistical significance of intergroup differences for categorical variables was assessed using the $\chi^{2}$ test. For continuous variables, a one-way ANOVA or the Kruskal-Wallis test was used. Odds ratios (ORs) for distinctive radiographic characteristics between OCSP subtypes were analyzed by multinomial logistic 
Table 1. Baseline characteristics

\begin{tabular}{lcccr}
\hline & TACI $(\mathrm{n}=69)$ & PACI $(\mathrm{n}=33)$ & LACI (n=59) & p value \\
\hline Mean age \pm SD, years & $69.2 \pm 10.3$ & $67.9 \pm 10.7$ & $66.1 \pm 10.2$ & 0.232 \\
Male & $40(58.0)$ & $17(51.5)$ & $35(59.3)$ & 0.756 \\
Median baseline NIHSS score (range) & $16(7-24)$ & $8(3-18)$ & $4(2-11)$ & $<0.001$ \\
Stroke risk factors & & & & \\
$\quad$ Hypertension & $39(56.5)$ & $19(57.6)$ & $41(69.5)$ & 0.283 \\
$\quad$ Diabetes & $16(23.2)$ & $12(36.4)$ & $20(33.9)$ & 0.273 \\
$\quad$ Dyslipidemia & $24(34.8)$ & $16(48.5)$ & $30(50.8)$ & 0.152 \\
$\quad$ Smoking & $17(24.6)$ & $10(30.3)$ & $18(30.5)$ & 0.719 \\
$\quad$ Atrial fibrillation & $34(49.3)$ & $13(39.4)$ & $7(11.9)$ & $<0.001$ \\
Median OTDT (range), min & $42.0(7-161)$ & $75.0(12-148)$ & $75.0(10-155)$ & 0.360 \\
Median DTNT (range), min & $66.0(18-155)$ & $68.0(40-102)$ & $66.0(33-175)$ & 0.870 \\
Median DTMT (range), min & $19.0(10-95)$ & $19.0(11-85)$ & $23.0(10-110)$ & 0.674 \\
Median NTMT (range), min & & &
\end{tabular}

Values are n (\%) except where otherwise indicated. OTDT = Onset-to-door time; DTNT = door-to-IV alteplase time; DTMT = door-to-MRI time; NTMT = IV alteplase-to-MRI time.

regression. Sensitivity, specificity, positive predictive value, and negative predictive value were calculated using standard formulas. In addition, 95\% confidence intervals (CIs) for these parameters were calculated according to the efficient score method. The results were considered statistically significant if $\mathrm{p}<0.05$.

\section{Results}

We screened a total of 174 eligible patients. Of these, 13 patients failed to complete the full MRI sequences, which included DWI, FLAIR, T2*-weighted gradient-echo imaging, 3D TOF-MRA, and PWI, because of excessive patient motion, cardiopulmonary compromise, contrast injection failure, or technologist error. Overall, 161 patients ( $42.9 \%$ women) with a mean age of $67.8 \pm 10.2$ years (median 69 years; range $34-88$ ) were included for analysis. The OCSP classification on admission showed 69 patients with TACI (42.9\%), 33 patients with PACI (20.5\%), and 59 patients with LACI $(36.6 \%)$. The median baseline NIHSS score in the OCSP subtypes was 16 (range 7-24) for TACI, 8 (3-18) for PACI, and 4 (2-11) for LACI ( $\mathrm{p}<$ $0.001)$. The etiologic stroke classification revealed 29 cases of large-artery atherosclerosis, 53 cases of cardioembolism, 33 cases of small-vessel occlusion, and 46 cases of undetermined etiology. Among stroke risk factors, atrial fibrillation was more prevalent in TACI $(49.3 \%)$ and PACI patients $(39.4 \%)$ than in LACI patients $(11.9 \%$; $p<0.001)$. The baseline characteristics of each OCSP subtype were summarized in table 1.

Thirty-four patients were treated with a $0.9-\mathrm{mg} / \mathrm{kg}$ dose of IV alteplase and 127 patients were treated with a $0.6-\mathrm{mg} / \mathrm{kg}$ dose of IV alteplase. MRI after a $0.9-\mathrm{mg} / \mathrm{kg}$ alteplase infusion revealed no occlusion in 22 patients, ICA occlusion in 3, MCA stem occlusion in 3, and MCA $\mathrm{M} 2$ occlusion in 6 patients. MRI after a $0.6-\mathrm{mg} / \mathrm{kg}$ alteplase infusion revealed no occlusion in 53 patients, ICA occlusion in 24, MCA stem occlusion in 26, and MCA M2 occlusion in 24 patients. The difference between the two dosage groups was not statistically significant $(\mathrm{p}=$ 0.082 ). 
Table 2. MRI parameters according to the OCSP subtypes

\begin{tabular}{|c|c|c|c|c|}
\hline & TACI $(n=69)$ & PACI $(n=33)$ & LACI $(n=59)$ & $\mathrm{p}$ value \\
\hline Arterial occlusion & $57(82.6)$ & $19(57.6)$ & $10(16.9)$ & $<0.001$ \\
\hline Site of arterial occlusion & & & & $<0.001$ \\
\hline ICA & $26(37.7)$ & $1(3.0)$ & $0(0)$ & \\
\hline MCA stem & $20(29.0)$ & $5(15.2)$ & $4(6.8)$ & \\
\hline MCA M2 & $11(15.9)$ & $13(39.4)$ & $6(10.2)$ & \\
\hline PWI-DWI mismatch & $40(58.0)$ & $25(75.8)$ & $20(33.9)$ & $<0.001$ \\
\hline Median ASPECTS $_{\text {DWI }}(\text { range })^{1}$ & $8(0-10)$ & $9(7-10)$ & $9(7-10)$ & $<0.001$ \\
\hline Median ASPECTS $_{\text {PWI }}$ (range) $^{1}$ & $5(0-10)$ & $8(3-10)$ & $9(4-10)$ & $<0.001$ \\
\hline ASPECTS $_{\text {DWI-PWI }} \geq 1$ & $43(62.3)$ & $23(74.2)$ & $19(33.3)$ & $<0.001$ \\
\hline ASPECTS $_{\text {DWI-PWI }} \geq 2$ & $35(50.7)$ & $11(35.5)$ & $7(12.3)$ & $<0.001$ \\
\hline ASPECTS $_{\text {DWI-PWI }} \geq 3$ & $26(37.7)$ & $5(16.1)$ & $4(7.0)$ & $<0.001$ \\
\hline HVS & $64(92.8)$ & $21(63.6)$ & $15(25.4)$ & $<0.001$ \\
\hline Microbleeds & $8(11.6)$ & $2(6.1)$ & $12(20.3)$ & 0.129 \\
\hline Leukoaraiosis & $31(44.9)$ & $20(60.6)$ & $32(54.2)$ & 0.291 \\
\hline Radiographic IA candidate & $30(43.5)$ & $6(18.2)$ & $4(6.8)$ & $<0.001$ \\
\hline Radiographic lacunar infarct & $1(1.4)$ & $1(3.0)$ & $32(54.2)$ & $<0.001$ \\
\hline
\end{tabular}

Values are n (\%) except where otherwise indicated.

${ }^{1}$ Two patients each (4 in total) in the PACI and LACI groups were excluded in the ASPECTS analysis because of isolated anterior cerebral artery territory infarcts.

MRI was performed for all screened patients either during or shortly after alteplase infusion. The median time from the start of IV alteplase to the start of MRI was 21 min (range 10-110). Patients were grouped according to two time intervals, those having MRI within 30 min from the start of alteplase, and those having MRI at least $30 \mathrm{~min}$ after alteplase. The presence of persistent major arterial occlusion (ICA or MCA stem) despite alteplase was not significantly different between the two groups, and was as follows: 35 (34.7\%) of 101 patients ( $\leq 30 \mathrm{~min}$ ) and $21(35.0 \%)$ of 60 patients ( $>30 \mathrm{~min}$ ), respectively, had persistent major occlusion despite alteplase $(\mathrm{p}=0.964)$.

In total, $40 / 161$ patients $(24.8 \%)$ were candidates for IA rescue reperfusion. Specifically, 30/69 TACI (43.5\%), 6/33 PACI (18.2\%), and 4/59 LACI patients (6.8\%) were candidates for IA rescue reperfusion $(\mathrm{p}<0.001)$. The predictive value for IA reperfusion candidates in the clinical TACI group showed $75.0 \%$ sensitivity ( $95 \%$ CI, 0.58-0.87), $67.8 \%$ specificity ( $95 \%$ CI, $0.59-0.76$ ), a positive predictive value of $43.5 \%$ ( $95 \%$ CI, $0.32-0.56)$, and a negative predictive value of $89.1 \%$ ( $95 \% \mathrm{CI}, 0.80-0.94)$.

With respect to the presence of major arterial occlusion, 56/161 patients (34.8\%) had a major arterial occlusion (ICA or MCA stem) despite IV alteplase. Relative to clinical OCSP, we found that 46/69 TACI (66.7\%), 6/33 PACI (18.2\%), and 4/59 LACI patients (6.8\%) had a major arterial occlusion despite IV alteplase $(\mathrm{p}<0.001)$.

With respect to penumbra, PWI-DWI mismatch was found in 85/161 patients $(52.8 \%)$. More specifically, PWI-DWI mismatch was found in 40/69 TACI (58.0\%), 25/33 PACI (75.8\%), and $20 / 59$ LACI patients $(33.9 \%)(p<0.001)$. However, this accounts only for a PWI-DWI mismatch greater than $20 \%$, and obscures the fact that TACI patients tend to have a larger penumbra area. Of the patients with ASPECTS $_{\text {DWI-PWI }} \geq 2,66.0 \%$ were classified as TACI patients $(\mathrm{p}<0.001)$, and of the patients with ASPECTS $_{\text {DWI-PWI }} \geq 3,74.3 \%$ were classified as TACI patients $(\mathrm{p}<0.001)$. 
Table 3. ORs (95\% CIs) for distinctive radiographic characteristics between the OCSP subtypes using multinomial regression analyses

\begin{tabular}{lclc}
\hline & TACI & PACI & LACI \\
\hline Arterial occlusion & $23.3(9.3-58.5)^{\mathrm{a}}$ & $6.7(2.5-17.5)^{\mathrm{a}}$ & 1.0 (ref.) \\
PWI-DWI mismatch & $2.7(1.3-5.5)^{\mathrm{b}}$ & $6.1(2.3-15.9)^{\mathrm{a}}$ & 1.0 (ref.) \\
Rescue IA candidate & $10.6(3.5-32.5)^{\mathrm{a}}$ & $3.1(0.8-11.7)^{\mathrm{c}}$ & 1.0 (ref.) \\
Lacunar infarct $^{\mathrm{c}}$ & 1.0 (ref.) $^{\mathrm{c}}$ & $2.2(0.1-35.1)^{\mathrm{c}}$ & $80.6(10.5-619.6)^{\mathrm{a}}$ \\
\hline
\end{tabular}

${ }^{\mathrm{a}} \mathrm{p}<0.001 ;{ }^{\mathrm{b}} \mathrm{p}<0.01 ;{ }^{\mathrm{c}} \mathrm{p}>0.05$

Among radiographic factors, HVS was found in 100 patients (62.1\%): 64/69 TACI (92.8\%), 21/33 PACI (63.6\%), and 15/59 LACI patients $(25.4 \%)(\mathrm{p}<0.001)$. The relationship between MRI findings and OCSP subtypes is summarized in table 2.

Multinomial logistic analysis on OCSP subtypes showed that persistent arterial occlusions were significantly more prevalent in the TACI (OR 23.3; 95\% CI, 9.3-58.5; $\mathrm{p}<0.001$ ) and PACI (OR 6.7; 95\% CI, 2.5-17.5; $\mathrm{p}<0.000$ ) groups than in the LACI group. Also, PWI-DWI mismatch was more prevalent in the clinical TACI and PACI groups, when compared to the LACI group. With respect to IA candidates, the clinical TACI group had a significantly higher predictive value, as compared to the LACI group (OR 10.6; 95\% CI, 3.5-32.5; p < 0.001). As expected, the radiographic lacunar infarcts were highly predicted by the clinical LACI group, when compared with the TACI group (OR 80.6; 95\% CI, 10.5-619.6; $\mathrm{p}<0.001$ ). Table 3 shows the ORs for distinctive radiographic characteristics between the OCSP subtypes.

\section{Discussion}

The neurological outcome following an ischemic stroke caused by occlusion of a large intracranial vessel is primarily dependent on timely recanalization [15]. During in-hospital stroke care, treatment time may be extended in a variety of ways, including time taken to decide on a particular treatment option and time taken to complete necessary preparation to execute that strategy [16]. Early information that increases the likelihood of choosing a particular treatment option, including, in this case, early anticipation of candidacy for IA rescue reperfusion, could reduce in-hospital delays by motivating early preparation for the execution of that treatment strategy. IA rescue reperfusion therapy as a treatment option is typically based on evidence of persistent major arterial occlusion and extended perfusiondiffusion mismatch. This study correlates baseline clinical OCSP subtypes with MRI of perfusion-diffusion mismatch and persistent major arterial occlusion. Since a baseline clinical OCSP classification is gatherable very early in the course of stroke management, clinical stroke subtypes can provide quick information about the likelihood of a patient needing IA rescue reperfusion therapy.

One of the key criteria to proceed with IA reperfusion therapy is the demonstration of extended penumbral area. This study is the first to examine the relationship between OCSP and PWI-DWI mismatch using MRI taken within $4.5 \mathrm{~h}$ of stroke onset. This is significant, since early MRI is necessary to accurately evaluate the penumbra. As such, this is the first study to assess connections between OCSP and IA reperfusion candidacy with all relevant criteria considered. The presence and volume of PWI-DWI mismatch can be affected by the site of arterial occlusion, the state of collateral circulation, the response to IV alteplase and the 
timing of MRI. In our study, the proportion of $20 \%$ mismatch was highest in the PACI group. However, the total area of hypoperfusion and mismatch, based on the ASPECT score, was largest in the TACI group. In considering IA rescue therapy, more brain parenchyma can be saved by reperfusion in the TACI group compared to the other groups, since the extent of ischemic penumbra is expected to be more sizable. This important topic requires further investigation.

Conflicting results have been previously reported regarding the relationship between OCSP classification subtypes and the presence of persistent vascular occlusion. One study found a positive correlation, whereas two other studies found no correlation [2,3,9]. However, these studies are limited by the fact that vascular examinations were based solely on findings from transcranial and carotid ultrasonography. In the present study, persistent major arterial occlusion (in the ICA or MCA stem) was highly prevalent in the TACI group (66.7\%), less prevalent in the PACI group (18.2\%), and even less prevalent in the LACI group (6.8\%). These results support a correlation found in a previous study between OCSP subtypes and intracranial arterial abnormalities detected on transcranial Doppler during acute stages of ischemic stroke [2]. In our study, the TACI group had by far the highest incidence of persistent major arterial occlusion. The low incidence of major arterial occlusion in the LACI group was thought to be explained by the association of lacunar infarction with small vessel occlusion [17]. However, 4 of 59 cases in the LACI group showed major arterial occlusion, suggesting that lacunar infarction cannot be determined with certainty using a clinical classification scheme during the hyperacute stage of an ischemic stroke.

Also, the persistence of arterial occlusion despite IV alteplase treatment may identify correlations between OCSP subtypes and alteplase 'non-responders'. The results above only demonstrate a link between OCSP subtypes and major arterial occlusion, as it is a key criterion for IA reperfusion therapy. However, the relationship between clinical subtypes and the persistence of any occlusion despite alteplase, more proximal or not, is of interest. A further important point, is the correlation of OCSP with the effectiveness of IV alteplase. Our result showed that the TACI group had the highest incidence of persistent vessel occlusion following alteplase. However, this result should be read cautiously in light of two limitations of this study regarding alteplase and the persistence of arterial occlusion $[18,19]$. First, different patients were given different doses of alteplase (either 0.6 or $0.9 \mathrm{mg} / \mathrm{kg}$ ), based on a judgment by the attending neurologist. Nevertheless, differences regarding the persistence of arterial occlusion between the two dosage groups were not statistically significant $(p=0.082)$. Second, a concern unique to this study was that MRI was taken relatively quickly after the start of alteplase, and thus it was uncertain whether there was enough time for the alteplase to work. One study examining the recanalization time window showed that alteplase-related recanalization occurred within the first hour of treatment [20]. Therefore, patients were grouped according to two time intervals, those having MRI within $30 \mathrm{~min}$ from the start of alteplase, and those having MRI at least $30 \mathrm{~min}$ after alteplase. The presence of persistent major arterial occlusion despite alteplase was not significantly different between the two groups $(\leq 30 \mathrm{~min}$ : 34.7\%; >30 min: 35.0\%; $\mathrm{p}=0.964$ ).

Finally, Schellinger et al. [21] reported that HVS could be a useful marker for accurate assessment of intracranial vessel status in hyperacute stroke patients. Consistent with that study, HVS on FLAIR imaging in our study revealed significant differences between OCSP subtypes, and occurred most commonly in the TACI subtype.

The current study has some limitations. First, the stroke MRI, including 3D TOF-MRA, was performed during or shortly after initiation of the IV alteplase infusion. As a result, the incidence of arterial occlusion reported for each OCSP subtype underestimates the true proportion at baseline, as in some cases, the alteplase dissolved the clots prior to the MRI. Likewise, the incidence of non-responders to alteplase might be overestimated; in some 
cases, the alteplase may have dissolved the clots after the MRI. Therefore, we cannot exclude the possibility that the relationship between OCSP, IV alteplase, and the presence of arterial occlusion might be overstated or understated. To mitigate this concern, we grouped patients according to two time intervals involving the start of alteplase infusion and the start of MRI and found no statistically significant difference. The second limitation of this study is that we used a simple visual assessment of mismatch on MRI to define the presence of salvageable ischemic tissue, so this may underestimate the true proportion of mismatch [22]. Finally, we used two different dosages of alteplase in this study ( 0.6 or $0.9 \mathrm{mg} / \mathrm{kg}$ ); therefore, the results should be read cautiously in terms of the presence of arterial occlusion, although the differences between the two doses were not statistically significant $[23,24]$.

In conclusion, the simple, quick, and bedside clinical OCSP classification in hyperacute ischemic stroke can play a useful role in predicting the likelihood of IA reperfusion candidacy. Specifically, patients classified as clinical TACI were significantly more likely to be candidates for IA rescue reperfusion $(\mathrm{p}<0.001)$. Additionally, TACI patients have a higher incidence of major arterial occlusion, and a penumbra area significantly larger than normal. Therefore, we propose to use the OCSP classification in hyperacute stroke as an 'early warning indicator' for IA reperfusion candidacy, which can allow for advanced preparation of IA therapy and theoretically shorten treatment time and reduce infarction.

\section{Acknowledgements}

The authors would like to thank Wade Martin of the Medical Research International for his critical English revision.

\section{References}

1 Bamford J, Sandercock P, Dennis M, Burn J, Warlow C: Classification and natural history of clinically identifiable subtypes of cerebral infarction. Lancet 1991;337:1521-1526.

-2 Mead GE, Wardlaw JM, Dennis MS, Lewis SC, Warlow CP: Relationship between pattern of intracranial artery abnormalities on transcranial Doppler and Oxfordshire Community Stroke Project clinical classification of ischemic stroke. Stroke 2000;31:714-719.

-3 Thomassen L, Waje-Andreassen U, Naess H, Aarseth J, Russell D: Combined carotid and transcranial ultrasound findings compared with clinical classification and stroke severity in acute ischemic stroke. Cerebrovasc Dis 2006;21:86-90.

4 Rubiera M, Ribo M, Pagola J, Coscojuela P, Rodriguez-Luna D, Maisterra O, et al: Bridging intravenous-intraarterial rescue strategy increases recanalization and the likelihood of a good outcome in nonresponder intravenous tissue plasminogen activator-treated patients: a case-control study. Stroke 2011;42:993-997.

-5 IMS Study Investigators: Combined intravenous and intra-arterial recanalization for acute ischemic stroke: the Interventional Management of Stroke Study. Stroke 2004;35:904-911.

6 Smith WS, Sung G, Starkman S, Saver JL, Kidwell CS, Gobin YP, et al: Safety and efficacy of mechanical embolectomy in acute ischemic stroke: results of the MERCI trial. Stroke 2005;36:1432-1438.

7 Shaltoni HM, Albright KC, Gonzales NR, Weir RU, Khaja AM, Sugg RM, et al: Is intra-arterial thrombolysis safe after full-dose intravenous recombinant tissue plasminogen activator for acute ischemic stroke? Stroke 2007; 38:80-84.

8 IMS II Trial Investigators: The Interventional Management of Stroke (IMS) II Study. Stroke 2007;38:21272135.

-9 Li H, Wong KS, Kay R: Relationship between the Oxfordshire Community Stroke Project classification and vascular abnormalities in patients with predominantly intracranial atherosclerosis. J Neurol Sci 2003;207: 65-69.

10 Kobayashi A, Wardlaw JM, Lindley RI, Lewis SC, Sandercock PAG, Czlonkowska A, et al: Oxfordshire community stroke project clinical stroke syndrome and appearances of tissue and vascular lesions on pretreatment ct in hyperacute ischemic stroke among the first 510 patients in the Third International Stroke Trial (IST-3). Stroke 2009;40:743-748. 
11 The National Institute of Neurological Disorders and Stroke rt-PA Stroke Study Group: Tissue plasminogen activator for acute ischemic stroke. N Engl J Med 1995;333:1581-1587.

12 Butcher K, Parsons M, Allport L, Lee SB, Barber PA, Tress B, et al: Rapid assessment of perfusion-diffusion mismatch. Stroke 2008;39:75-81.

13 Röther J, Schwartz A, Wentz KU, Rautenberg W, Hennerici M: Middle cerebral artery stenoses: assessment by magnetic resonance angiography and transcranial Doppler ultrasound. Cerebrovasc Dis 1994;4:273-279.

14 Kamran S, Bates V, Bakshi R, Wright P, Kinkel W, Miletich R: Significance of hyperintense vessels on FLAIR MRI in acute stroke. Neurology 2000;55:265-269.

15 Rha JH, Saver JL: The impact of recanalization on ischemic stroke outcome: a meta-analysis. Stroke 2007;38: 967-973.

16 Evenson KR, Foraker RE, Morris DL, Rosamond WD: A comprehensive review of prehospital and in-hospital delay times in acute stroke care. Int J Stroke 2009;4:187-199.

17 Rajajee V, Kidwell C, Starkman S, Ovbiagele B, Alger J, Villablanca P, et al: Diagnosis of lacunar infarcts within $6 \mathrm{~h}$ of onset by clinical and CT criteria versus MRI. J Neuroimaging 2008;18:66-72.

18 Saqqur M, Uchino K, Demchuk AM, Molina CA, Garami Z, Calleja S, et al: Site of arterial occlusion identified by transcranial Doppler predicts the response to intravenous thrombolysis for stroke. Stroke 2007;38:948-954.

19 Linfante I, Llinas RH, Selim M, Chaves C, Kumar S, Parker RA, et al: Clinical and vascular outcome in internal carotid artery versus middle cerebral artery occlusions after intravenous tissue plasminogen activator. Stroke 2002;33:2066-2071.

20 Ribo M, Alvarez-Sabín J, Montaner J, Romero F, Delgado P, Rubiera M, et al: Temporal profile of recanalization after intravenous tissue plasminogen activator: selecting patients for rescue reperfusion techniques. Stroke 2006;37:1000-1004.

-21 Schellinger PD, Chalela JA, Kang DW, Latour LL, Warach S: Diagnostic and prognostic value of early MR Imaging vessel signs in hyperacute stroke patients imaged. AJNR Am J Neuroradiol 2005;26:618-624.

22 Campbell BCV, Christensen S, Foster SJ, Desmond PM, Parsons MW, Butcher KS, et al: Visual assessment of perfusion-diffusion mismatch is inadequate to select patients for thrombolysis. Cerebrovasc Dis 2010;29: 592-596.

23 Yamaguchi T, Mori E, Minematsu K, Nakagawara J, Hashi K, Saito I, et al: Alteplase at $0.6 \mathrm{mg} / \mathrm{kg}$ for acute ischemic stroke within 3 hours of onset: Japan Alteplase Clinical Trial (J-ACT). Stroke 2006;37:1810-1815.

24 Mori E, Minematsu K, Nakagawara J, Yamaguchi T, Sasaki M, Hirano T, et al: Effects of $0.6 \mathrm{mg} / \mathrm{kg}$ intravenous alteplase on vascular and clinical outcomes in middle cerebral artery occlusion: Japan Alteplase Clinical Trial II (J-ACT II). Stroke 2010;41:461-465. 